Ergod. Th. \& Dynam. Sys. (1985), 5, 587-593

Printed in Great Britain

\title{
An Anosov action on the bundle of Weyl chambers
}

\author{
HANS-CHRISTOPH IM HOF \\ Mathematisches Institut der Universität Basel, \\ Rheinsprung 21, CH-4051 Basel, Switzerland
}

(Received 15 October 1984 and revised 1 March 1985)

\begin{abstract}
We introduce an Anosov action on the bundle of Weyl chambers of a riemannian symmetric space of non-compact type, which for rank one spaces coincides with the geodesic flow.
\end{abstract}

\section{Introduction}

The geodesic flow of a riemmanian symmetric space of non-compact type is an Anosov flow if and only if the rank of the space is one. In this note we shall introduce an action, which for spaces of rank one coincides with the geodesic flow, but which is Anosov for all riemannian symmetric spaces of non-compact type. The basic idea in defining this action is to view geodesics not as particular curves of the space, but as totally geodesic flat subspaces. So we are led to consider flats, i.e. maximal totally geodesic flat subspaces, and Weyl chambers as particular subsets of flats. The bundle of all Weyl chambers will be the phase space of the action. The geodesic flow is given by moving geodesic rays along their supporting geodesics. Similarly, the action to be defined here consists of parallel translating Weyl chambers within their supporting flats. If the rank of the space is one, then flats are geodesics, Weyl chambers are geodesic rays, and our action coincides with the geodesic flow. For spaces of rank higher than one, i.e. spaces with higher-dimensional flats, this action is no longer a flow, but an action of a higher-dimensional abelian Lie group.

Let us recall the definition of an Anosov action (cf. [5]; for the ergodicity of Anosov actions, see [8]). An action $\varphi$ of a Lie group $L$ on a riemannian manifold $M$ is said to be Anosov if the orbits under $\varphi$ foliate $M$, and if there exists at least one Anosov element in $L$, i.e. an element $a \in L$ such that the tangent bundle $T(M)$ admits a $d \varphi_{a}$-invariant splitting

$$
T(M)=E^{s} \oplus E^{c} \oplus E^{u}
$$

into three sub-bundles: the central bundle $E^{c}$ tangent to the orbits under $\varphi$, the strong stable bundle $E^{s}$, on which the differential $d \varphi_{a}$ contracts more than on $E^{c}$, and the strong unstable bundle $E^{u}$, on which $d \varphi_{a}$ expands more than on $E^{c}$.

Given such a splitting, the stable and unstable bundles are defined to be the sums $E^{s} \oplus E^{c}$ and $E^{u} \oplus E^{c}$, respectively. Finally, the foliations of $T(M)$ tangent to $E^{s}$, $E^{u}, E^{s} \oplus E^{c}$, and $E^{u} \oplus E^{c}$ are called respectively the strong stable, strong unstable, stable, and unstable foliation induced by $\varphi_{a}$. 
The first part of this note is purely group-theoretical. There we shall define our action and prove its Anosov character. Geometrical interpretations will be given in the second part, where we shall introduce the bundle of Weyl chambers and relate our action to the geodesic flow. Finally the stable and unstable foliations will find an interpretation in terms of horocycles.

The content of this note is part of the author's Habilitationsschrift [6]. I wish to thank Ernst Ruh and Mikhael Gromov for their encouragement and criticism. For financial support I am indebted to the Sonderforschungsbereich 'Theoretische Mathematik' at the University of Bonn, the Stiftung Volkswagenwerk, and the IHES in Bures-sur-Yvette, France.

\section{The action of $A$ on $G / M$}

Here we state and prove our main result in group-theoretical terms. Let $G$ be a real semisimple Lie group of non-compact type and finite centre. A maximal compact subgroup $K \subset G$ gives rise to a Cartan decomposition $g=k \oplus h$, where $g$ and $k$ denote the respective Lie algebras of $G$ and $K$, and $\not$ is the orthogonal complement to $k$ with respect to the Killing form on $g$. Let $a \subset h$ be a maximal abelian subalgebra and $A=\exp a \subset G$ the corresponding subgroup. By log: $A \rightarrow a$ we shall denote the inverse of exp: $a \rightarrow A$. The pair $(g, a)$ gives rise to a root system $\Lambda \subset \operatorname{Hom}(a, \mathbb{R})$ and a root space decomposition

$$
g=g_{0} \oplus \sum_{\lambda \in \Lambda} g_{\lambda}
$$

An element $a \in A$ is called regular if $\lambda(\log a) \neq 0$ for all $\lambda \in \Lambda$, otherwise it is called singular. Now consider $M=Z_{K}(A)$, the centralizer of $A$ in $K$. By defining $\varphi(a, g M)=\varphi_{a}(g M)=g a M$ we obtain an action $\varphi$ of $A$ on the homogeneous space $G / M$.

Theorem. The action $\varphi: A \times G / M \rightarrow G / M$ defined by $\varphi(a, g M)=g a M$ is an Anosov action.

Proof. The orbits under $\varphi$ coincide with the fibres of the natural projection $G / M \rightarrow$ $G / M A$, therefore they are the leaves of a foliation. Now we shall prove that all regular elements of $\boldsymbol{A}$ are Anosov elements for $\varphi$. At first we fix an arbitrary $a \in A$. We have to compute the differential of

$$
\varphi_{a}: G / M \rightarrow G / M
$$

at a point $g M \in G / M$. Let $m$ denote the Lie algebra of $M$. Since all tangent spaces of $G / M$ are identified with $g / m$ by left translation, it suffices to compute

$$
d \varphi_{a}: g / m \rightarrow g / m \text {. }
$$

Let $R_{a}: G \rightarrow G$ denote right translation by $a$. The projection of $R_{a}$ onto $G / M$ is just $\varphi_{a}$. The differential of $R_{a}$ is $\operatorname{Ad}\left(\mathrm{a}^{-1}\right): g \rightarrow g$, and this in turn projects onto $\mathrm{g} / \mathrm{m}$. Let us denote this projection again by $\operatorname{Ad}\left(a^{-1}\right)$, then we have

$$
d \varphi_{a}=\operatorname{Ad}\left(a^{-1}\right): g / m \rightarrow g / m \text {. }
$$

In order to compute $\operatorname{Ad}\left(a^{-1}\right)$ we use the root space decomposition of $g$ with respect 
to $a$. Observing $g_{0}=m \oplus a$, we have

therefore

$$
g=m \oplus a \oplus \sum_{\lambda \in \Lambda} g_{\lambda}
$$

$$
g / m \cong a \oplus \sum_{\lambda \in \Lambda} g_{\lambda} .
$$

According to this isomorphism an element $\xi \in g / m$ will be written as

$$
\xi=\xi_{0}+\sum_{\lambda \in \Lambda} \xi_{\lambda}
$$

with $\xi_{0} \in a$ and $\xi_{\lambda} \in g_{\lambda}$. The equation

$$
\operatorname{Ad}\left(a^{-1}\right)=\exp (\operatorname{ad}(-\log a))
$$

in GL $(g)$ implies

$$
\operatorname{Ad}\left(a^{-1}\right)(\xi)=\xi_{0}+\sum_{\lambda \in \Lambda} e^{-\lambda(\log a)} \xi_{\lambda}
$$

for $\xi$ as given by (1).

Assume now that $a$ is regular. Then $\Lambda$ splits into the two subsets

$$
\left.\Lambda^{+}=\{\lambda \in \Lambda ; \lambda(\log a)>0\}, \quad \Lambda^{-}=\{\lambda \in \Lambda ; \lambda \log a)<0\right\} .
$$

Defining

we obtain

$$
x^{+}=\sum_{\lambda \in \Lambda^{+}} g_{\lambda}, \quad n^{-}=\sum_{\lambda \in \Lambda^{-}} g_{\lambda}
$$

$$
g / m=a \oplus n^{+} \oplus n^{-} \text {. }
$$

We claim that this decomposition, extended to all tangent spaces of $G / M$ by left translation, induces the desired splitting of $T(G / M)$. Indeed, $a$ is tangent to the orbits of $\varphi$, and by (2) we get

$$
\begin{aligned}
& d \varphi_{a}(\xi)=\xi \quad \in a, \\
& d \varphi_{a}(\xi)=\sum_{\lambda \in \Lambda^{+}} e^{-\lambda(\log a)} \xi_{\lambda} \quad \in n^{+}, \\
& d \varphi_{a}(\xi)=\sum_{\lambda \in \Lambda^{-}} e^{-\lambda(\log a)} \xi_{\lambda} \in n^{-},
\end{aligned}
$$

for $\xi \in a, \xi=\sum_{\lambda \in \Lambda^{+}} \xi_{\lambda} \in n^{+}, \xi=\sum_{\lambda \in \Lambda^{-}} \xi_{\lambda} \in n^{-}$, respectively. Thus (3) is invariant under $d \varphi_{a}$.

In order to establish the necessary estimates we need a metric on $G / M$. Inverting the sign of the Killing form on $k$ we get a positive definite scalar product on $g$ which we restrict onto $g / m \cong a \oplus \sum_{\lambda \in \Lambda} g_{\lambda}$. By left translation this induces a riemannian metric on $G / M$. Since root spaces are orthogonal to each other, we have

$$
\|\xi\|^{2}=\left\|\xi_{0}\right\|^{2}+\sum_{\lambda \in \Lambda}\left\|\xi_{\lambda}\right\|^{2}
$$

for $\xi$ as given by (1). Therefore our expressions for $d \varphi_{a}(\xi)$ imply

$$
\begin{aligned}
& \left\|d \varphi_{a}(\xi)\right\|=\|\xi\| \quad \text { for } \xi \in a ; \\
& \left\|d \varphi_{a}(\xi)\right\| \leq e^{-k}\|\xi\| \quad \text { for } \xi \in n^{+} \text {; } \\
& \left\|d \varphi_{a}(\xi)\right\| \geq e^{k}\|\xi\| \quad \text { for } \xi \in n^{-} ;
\end{aligned}
$$

where $k=\min \left\{\lambda(\log a) ; \lambda \in \Lambda^{+}\right\}$is a positive constant depending only on $a$. 
Remark. The same arguments applied to a singular element $a \in A$ lead to the decomposition

$$
g / m=\left(a \oplus \sum_{\lambda(\log a)=0} g_{\lambda}\right) \oplus \sum_{\lambda(\log a)>0} g_{\lambda} \oplus \sum_{\lambda(\log a)<0} g_{\lambda},
$$

which exhibits a component transverse to $a$, where $d \varphi_{a}$ is neither contracting nor expanding. Therefore singular elements of $\boldsymbol{A}$ are not Anosov elements for the action $\varphi$.

\section{Geometric interpretations}

Here we give geometric interpretations of the action $\varphi: A \times G / M \rightarrow G / M$ and of the stable and unstable foliations induced by this action. Let $X$ be a riemannian symmetric space of non-compact type. The maximal totally geodesic flat subspaces of $X$ are called flats. The common dimension of all flats of $X$ is called the rank of $X$. Each geodesic is contained in at least one flat. A geodesic is called regular (singular), if it is contained in exactly one flat (in more than one flat). We fix a point $x \in X$ and a flat $F \subset X$ with $x \in F$. The singular geodesics in $F$ through $x$, are contained in the union of finitely many hyperplanes in $F$ through $x$, and conversely, any geodesic lying in one of these hyperplanes is singular. The connected components of $F$ after removing all singular geodesics through $x$ are called the Weyl chambers of $F$ based at $x$. Admitting all possible flats $F \subset X$ and all possible base points $x \in F$, we obtain the set $C X$ of all Weyl chambers of $X$. Let $\pi: C X \rightarrow X$ denote the map which assigns to each Weyl chamber its base point. Following Mostow ([7, $\S 4]$ ) we call two Weyl chambers asymptotic, if each one lies in a tubular neighbourhood of the other. The set of classes of asymptotic Weyl chambers is called the boundary of $X$. Let $\tau$ denote the map which assigns to each Weyl chamber its asymptoticity class.

A flat with a distinguished class of asymptotic Weyl chambers is called an oriented flat. Let $\rho$ denote the map which assigns to a Weyl chamber $c$ its supporting flat together with the orientation induced by $c$.

Finally we shall sketch a geometric definition of horocycles (for more details see [6]). Each geodesic ray in $X$ determines a horosphere passing through the initial point of the ray and orthogonal to it (cf. [1, §3]). For a fixed Weyl chamber $c$ based at $x$, the horocycle associated with $c$ is defined as the intersection of all horospheres determined by the (regular) geodesic rays emanating from $x$ and contained in $c$. This intersection is a submanifold whose codimension equals the rank of $X$. Let $\sigma$ denote the map which assigns to each Weyl chamber its associated horocycle.

Now we shall translate the geometrical picture introduced so far into the grouptheoretical language. Let $G$ be the connected component of the isometry group of $X$; it is a real semisimple Lie group of non-compact type and trivial centre. Choose a point $x_{0} \in X$ and let $K$ be the isotropy group of $x_{0}$. Then $K$ is a maximal compact subgroup of $G$, and $X$ is identified with the homogeneous space $G / K$. Furthermore, the choice of $x_{0}$ gives rise to a Cartan decomposition $g=k \oplus h$, where the vector part $h$ is identified with the tangent space of $X$ at $x_{0}$. 
Now consider a flat $F$ through $x_{0}$. Its tangent space at $x_{0}$ is a maximal abelian subalgebra $a \subset h$, and conversely, each such subalgebra corresponds to a flat through $x_{0}$. The group $K$ operates transitively on the set of all flats through $x_{0}$ (cf. [3, Chapter $\mathrm{V}$, Lemma 6.3]).

We now fix a flat $F_{0}$ through $x_{0}$. Let $a$ denote the corresponding maximal abelian subalgebra of $\mu$, and set $A=\exp a \subset G$. The group $A$ acts on $F_{0}$ as its group of translations. The root system $\Lambda$ with respect to the pair $(g, a)$ defines Weyl chambers in $a$ and, via the exponential map, in $A$. These correspond to the Weyl chambers in $F_{0}$ based at $x_{0}$. The Weyl group $W$ of $X$ is defined as the quotient $M^{\prime} / M$, where $M=Z_{K}(A)$ is the centralizer and $M^{\prime}=N_{K}(A)$ the normalizer of $A$ in $K$. It acts simply transitively on the set of Weyl chambers in $F_{0}$ based at $x_{0}$ (for spaces of compact type, see [3, Chapter VII, Theorem 2.12]). Let $c_{0}$ be one of the Weyl chambers in $F_{0}$ based at $x_{0}$. The choice of $c_{0}$ determines a set $\Lambda^{+} \subset \Lambda$ of positive roots. Define $n=\sum_{\lambda \in \Lambda^{+}} g_{\lambda}$ and $N=\exp n \subset G$. Finally define $P=M A N$.

The Weyl chamber $c_{0}$ and the groups derived from this choice shall be fixed for the rest of this paper.

Proposition. Let $X$ be a riemannian symmetric space of non-compact type. Then the set of Weyl chambers of $X$ is identified with the homogeneous space $G / M$, the set of oriented flats with $G / M A$, the set of horocycles with $G / M N$, and the boundary of $X$ with $G / P$. Furthermore, the maps $\pi, \rho, \sigma$, and $\tau$ introduced above coincide with the natural projections from $G / M$ to $G / K, G / M A, G / M N$, and $G / P$, respectively.

Proof. We shall give a proof for the case of $G / M$. First we have to show that $G$ operates transitively on $C X$. Let $c$ be any Weyl chamber, $F$ the flat containing $c$, and $x$ the base point of $c$. An isometry $g \in G$ takes the flat $F$ into a flat $g F$ through the point $g x$, and it preserves the configuration of regular and singular geodesics. Therefore the Weyl chamber $c$ is mapped into a Weyl chamber $g c$ contained in $g F$ and based at $g x$.

Now we shall find an isometry which takes $c$ into $c_{0}$. There is a $g \in G$ such that $g x=x_{0}$, thus $x_{0} \in g F$. An element $k \in K$ maps $g F$ into $F_{0}$, hence $k g F=F_{0}$. Now $k g c$ and $c_{0}$ are two Weyl chambers in $F_{0}$ based at $x_{0}$, therefore there is an element $m^{\prime} \in M^{\prime}$ such that $m^{\prime} k g c=c_{0}$.

Finally we shall determine the isotropy group of $c_{0}$ in $G$. Suppose $g c_{0}=c_{0}$. Then certainly $g x_{0}=x_{0}$, hence $g \in K$. Moreover $g F_{0}=F_{0}$, thus $g \in M^{\prime}$. Since $W=M^{\prime} / M$ acts simply transitively on the set of Weyl chambers in $F_{0}$ based at $x_{0}$, it follows that $g \in M$. Conversely, we clearly have $m c_{0}=c_{0}$ for $m \in M$.

The proof for $G / M A$ is similar to the one for $G / M$, so we shall omit it.

The case of $G / M N$ is treated in [4] in purely group-theoretical terms. There, the horocycle associated with $c=g c_{0}$ is defined as the orbit of $g x_{0}$ under the group $g \mathrm{Ng}^{-1}$. The relation with our geometric notion of horocycles is established in [6].

The identification of the boundary $G / P$ with the set of classes of asymptotic Weyl chambers is proved in $[7, \S 4]$.

The statements about the natural projections are evident. 
In addition to the obvious left action of $G$ on the spaces $G / M, G / M A, G / M N$, and $G / P$, there is a right action of the Weyl group $W$ on these spaces. Let $m_{w}$ denote a representative for an element $w \in W$. Then the effect of $w$ on a Weyl chamber $g M$ is given by $g M \mapsto g m_{w} M$. The action of $W$ on the other spaces is defined similarly, and so all these $W$-actions are compatible with natural projections.

Now recall the action $\varphi: A \times G / M \rightarrow G / M$ given by $\varphi(a, g M)=g a M$. The ortit of a Weyl chamber $g M \in G / M$ under $\varphi$ is $\{g a M \in G / M ; a \in A\}$, it coincides with the fibre $\rho^{-1}(g M A)$ of the projection $\rho: G / M \rightarrow G / M A$. In other words, the effect of $\varphi$ consists of translating a given Weyl chamber along its supporting flat to another Weyl chamber of the same flat and asymptotic to the given one. In the rank one case, Weyl chambers coincide with geodesic rays, and these in turn may be identified with unit tangent vectors. Moreover, since the group $A$ is one-dimensional it admits a parametrization $A=\{a(t) \in G ; t \in \mathbb{R}\}$. Therefore the action $\varphi$ may be viewed as an action of $\mathbb{R}$ on the unit tangent bundle of $X$. The effect of $\varphi$ consists in this case of translating a given geodesic ray along its supporting geodesic to another ray of the same geodesic and pointing to the same direction. This is exactly the effect of the geodesic flow. (For a group-theoretical description of the geodesic ftow, see [2].)

Finally we shall determine the leaves of the stable and strong stable foliations induced by $\varphi$. First we shall consider a special case. Let $a_{0}$ be a regular element of $A$ with $\lambda\left(\log a_{0}\right)>0$ for all $\lambda \in \Lambda^{+}$. For such an element, the contracting subspace of $g / m \cong a \oplus \sum_{\lambda \in \Lambda} g_{\lambda}$ is $n=\sum_{\lambda \in \Lambda^{+}} g_{\lambda}$. Therefore the leaf of the strong stable foliation through $c_{0}=M \in G / M$ equals the orbit

$$
\{n M \in G / M ; n \in N\}
$$

of $c_{0}$ under $N$. This orbit coincides with the fibre $\sigma^{-1}(M N)$ of the projection $\sigma: G / M \rightarrow G / M N$, i.e. it consists of all Weyl chambers asymptotic to $c_{0}$ and based at the points of the horocycle $Z_{0}=N x_{0}=M N \in G / M N$.

The stable foliation is tangent to $a \oplus n$. Its leaf through $c_{0}$ equals the orbit

$$
\{a n M \in G / M ; a \in A, n \in N\}
$$

of $c_{0}$ under $A N$, it coincides with the fibre $\tau^{-1}(P)$ of the projection $\tau: G / M \rightarrow G / P$, i.e. it consists of all Weyl chambers asymptotic to $c_{0}$.

Now consider the case of an arbitrary regular element $a \in A$. There is a unique Weyl group element $w \in W$ such that $a=m_{w} a_{0} m_{w}^{-1}$, where $m_{w} \in M^{\prime}$ represents $w$, and $a_{0}$ satisfies $\lambda\left(\log a_{0}\right)>0$ for all $\lambda \in \Lambda^{+}$. The contracting subspace with respect to the action of $a$ is

$$
\sum_{\lambda(\log a)>0} g_{\lambda}=\operatorname{Ad}\left(m_{w}\right) n
$$

Therefore the leaf of the strong stable foliation through $c_{0}$ equals the orbit

$$
\left\{m_{w} n m_{w}^{-1} M \in G / M ; n \in N\right\}
$$

of $c_{0}$ under $m_{w} N m_{w}^{-1}$. This is no longer a set of asymptotic Weyl chambers, it is obtained from the set $\left\{m_{w} n M \in G / M ; n \in N\right\}$ of Weyl chambers asymptotic to $m_{w} c_{0}$ and based at the points of the horocycle $m_{w} Z_{0}$ by applying $w^{-1}$ from the right. 
The leaf of the stable foliation through $c_{0}$ equals the orbit

$$
\left\{m_{w} a n m_{w}^{-1} M \in G / M ; a \in A, n \in N\right\}
$$

of $c_{0}$ under $m_{w} A N m_{w}^{-1}=A m_{w} N m_{w}^{-1}$. It consists of the right $w^{-1}$-images of all Weyl chambers asymptotic to $m_{w} c_{0}$.

The unstable foliations with respect to $a$ coincide with the stable foliations with respect to $a^{-1}$, so they need no further attention.

\section{REFERENCES}

[1] P. Eberlein \& B. O'Neill. Y/isibility manifolds. Pacific J. Math. 46 (1973), 45-109.

[2] I. M. Gel'fand \& S. V. Fomin. Geodesic flows on manifolds of constant negative curvature. Uspehi Mat. Nauk (N.S.) 7 (1952), 118-137; Amer. Math. Soc. Translations (2) 1 (1955), 49-65.

[3] S. Helgason. Differential Geometry and Symmetric Spaces. Academic Press: New York 1962.

[4] S. Helgason. Duality and Radon transform for symmetric spaces. Amer. J. Math. 85 (1963), 667-692.

[5] M. W. Hirsch. Foliations and non-compact transformation groups. Bull. Amer. Math. Soc. 76 (1970), 1020-1023.

[6] H. C. Im Hof. Die Geometrie der Weylkammern in symmetrischen Räumen vom nichtkompakten Typ. Habilitationsschrift, Bonn, 1979.

[7] G. D. Mostow. Strong Rigidity of Locally Symmetric Spaces. Princeton University Press: Princeton, 1973.

[8] C. Pugh \& M. Shub. Ergodicity of Ansov actions. Invent. Math. 15 (1972), 1-23. 